\title{
Synthetic Long HPV16 E6/E7 Peptides Vaccine ISA101b
}

\author{
National Cancer Institute
}

\section{Source}

National Cancer Institute. Synthetic Long HPV16 E6/E7 Peptides Vaccine ISA101b. NCI

Thesaurus. Code C150809.

A therapeutic peptide vaccine consisting of nine overlapping synthetic long peptides (SLPs), 25 to 32 amino acids in size, derived from the human papillomavirus (HPV) type 16 (HPV16) oncoprotein E6 and three SLPs, each 35 amino acids in size, derived from HPV16 E7, with potential immunostimulating and antineoplastic activities. Upon subcutaneous administration, the synthetic long HPV16 E6/E7 peptides vaccine ISA101 b is taken up and the long peptides are proteolytically degraded to form shorter peptide epitopes by dendritic cells (DCs). The processed viral epitopes are presented by DCs, which stimulate the host immune system to mount helper T-cell and cytotoxic Tlymphocyte (CTL) responses against HPV16 E6/E7-expressing tumor cells. This results in the destruction of tumor cells and leads to decreased tumor growth. The HPV16 E6 and E7 oncoproteins are implicated in the tumorigenesis of a variety of cancers. The SLPS allow optimal presentation by antigen-presenting cells (APCS). Compared to ISA101, the E6 SLPs in ISA101 b are identical, overlap by ten to eighteen residues and cover the complete sequence of HPV16 E6. However, ISA101 has four overlapping SLPs E7 peptides while ISA101b has three E7 SLPs, which leaves seven amino acids of the E7 SLPs uncovered in ISA101 b but this modification is not likely to alter the immunogenicity of ISA101b. 